\title{
Escenario sobre la aplicación de Aprendizaje Ubicuo en un Entorno Activo
}

\section{Stage about the application of Ubiquitous-Learning in an Active Environment}

\author{
ARREDONDO-SALCEDO, Daniel $\dagger^{*}$, ESQUIVEL-SALAS, Abraham, SALAS-GUZMÁN, Manuel \\ Ignacio y CASTANEDDA-DELGADO, Jaime
}

Instituto Tecnológico Superior Zacatecas Norte, Departamento de Informática y Sistemas Computacionales

ID $1^{\mathrm{er}}$ Autor: Daniel, Arredondo-Salcedo / ORC ID: 0000-0003-3236-4880, CVU CONACYT ID: 316030

ID $1^{\mathrm{er}}$ Coautor: Abraham, Esquivel-Salas / ORC ID: 0000-0001-8258-8837

ID $2^{\text {do }}$ Coautor: Manuel Ignacio, Salas-Guzmán / ORC ID: 0000-0002-7039-2703, CVU CONACYT ID: 316159

ID $3{ }^{\text {er }}$ Coautor: Jaime, Castañeda-Delgado / ORC ID: 0000-0001-9786-4128, CVU CONACYT ID: 252889

DOI: $10.35429 /$ JTAE.2019.9.3.17.26

Recibido: 09 de Julio, 2019; Aceptado 30 de Septiembre, 2019

\begin{abstract}
Resumen
Ubiquitous-Learning y sus aplicaciones sensibles al contexto (context-aware) tiene el potencial de proporcionar un aprendizaje personalizado y activo, además de permitir al estudiante desarrollar actitudes y aptitudes a partir de la resolución de problemas, seguimiento del progreso, información, intercambio de conocimientos y proceso de colaboración. Nuestra contribución consiste en la creación de un entorno de aprendizaje ubicuo, que a partir de una serie de interfaces multimodales desarrolladas para un Entorno de Inteligencia Ambiental y aplicaciones sensibles al contexto, pretende incidir en actividades de enseñanzaaprendizaje a través de un laboratorio de prácticas colaborativas. El objetivo de la propuesta consiste en implementar el concepto de Aprendizaje Ubicuo (Ubiquitous Learning), dotando al estudiante de un espacio propicio, para generar conocimientos e incentivando el desarrollo de habilidades cognitivas. En este artículo, se hace una descripción de un escenario en el uso de la tecnología disponible en un Entorno Activo aplicada al mejoramiento del desempeño académico de algunas materias, en donde la nota de evaluación del alumno está relacionada directamente con sus habilidades prácticas, es decir, "el saber hacer".
\end{abstract}

Ubicuo, Aprendizaje, Entorno

\begin{abstract}
Ubiquitous-Learning and its context sensitive applications (context-aware) has the potential to provide personalized and active learning, in addition to allowing the student to develop attitudes and aptitudes based on problem solving, progress monitoring, information, exchange of information, knowledge and collaboration process. Our contribution consists of the creation of a ubiquitous learning environment, which from a series of multimodal interfaces developed for an Environment Intelligence Environment and context sensitive applications, aims to influence teaching-learning activities through a laboratory of practices collaborative. The objective of the proposal is to implement the concept of Ubiquitous Learning (Ubiquitous Learning), providing the student with a space conducive, to generating knowledge and encouraging the development of cognitive skills. In this article, a description about the stage of the available technology in an Active Environment applied to the improvement of the academic performance of some subjects, where the student's evaluation is relate to their practical skills, that is, "The know-how".
\end{abstract}

Ubiquitous, Learning, Environment

Citación: ARREDONDO-SALCEDO, Daniel, ESQUIVEL-SALAS, Abraham, SALAS-GUZMÁN, Manuel Ignacio y CASTAÑEDA-DELGADO, Jaime. Escenario sobre la aplicación de Aprendizaje Ubicuo en un Entorno Activo. Revista de Tecnología y Educación. 2019. 3-8: 17-26

$\uparrow$ Investigador contribuyendo como primer autor. 


\section{Introducción}

Entendemos por aprendizaje ubicuo $(u$ learning) como un entorno de aprendizaje de actividades cotidianas cuyo valor agregado es el uso de dispositivos móviles, computadoras embebidas y redes inalámbricas (Ogata et al,. 2009). Existen términos integrados al $u$ learning, como lo es m-learning, que apoya el aprendizaje mediante el uso de dispositivos portátiles como tablets, PDA y teléfonos celulares (Smartphone), el p-learning, en el cual pequeños dispositivos pueden obtener información del entorno de aprendizaje y reaccionar en consecuencia de dicha información, y finalmente el e-learning, el cual consiste en procesos de enseñanza -aprendizaje a través de internet caracterizados por una separación física entre instructor y estudiante mediante la comunicación digital.

Por otra parte, (Ogata and Yano, 2004) analiza el concepto USCL (Computer Supported Ubiquitous Learning) que consiste en un entorno de soporte a las actividades $u$ learnig que requieren un alto grado en embebimiento y movilidad. De esta manera, el embebimiento es provisto por los entornos activos que son un espacio físico potencializado con computadoras, redes de comunicación, sensores y actuadores para responder cotidianamente a las acciones, preferencias y necesidades de las personas, y por otra parte, la movilidad es provista por las plataformas virtuales y aplicaciones móviles accesibles a través de dispositivos portátiles (teléfonos, tabletas, PDA).

En una revisión sistemática sobre 176 estudios relacionados con $u$-learning (Cárdenas and Peña, 2018) se identificó la tendencia de que los trabajos en dicho ámbito se incrementaron al doble desde el 2014 y la generación de una clasificación cada vez más amplia de características, lo cual implica mayor productividad y madurez.

Es por eso que aprovechar las bondades del u-learning son un área de oportunidad para la formación de profesionistas competentes, mediante la promoción del intercambio de ideas para aprender de diversas maneras como la cooperación, colaboración e interacción social.
En el sistema tecnológico, regido por el Tecnológico Nacional de México (TecNM), la evaluación de asignaturas bajo el modelo de competencia siglo XXI (Acosta and Armendáriz, 2012) se realiza basado en tres tipos de contenido: conceptual, procedimental y actitudinal, en los cuales se valora la ejecución de las actividades de aprendizaje. Derivado de lo anterior existe una oportunidad de apoyarse de herramientas tecnológicas tanto para el desarrollo como la evaluación de actividades de aprendizaje. Por parte institucional, existe la necesidad de brindar evaluaciones y retroalimentaciones objetivas hacia los estudiantes, usando tecnología se busca promover el aprendizaje y su evaluación correspondiente de manera eficiente, evidenciable y oportuna.

El objetivo del presente artículo es hacer una descripción de un escenario en el uso de la tecnología disponible en un Entorno Activo aplicada al mejoramiento del desempeño académico de algunas materias, enfocándose en la realización y evaluación de las actividades $u$ learning en los rubros de desempeño y actitud de los estudiantes.

La retroalimentación a partir de la evaluación formativa, tiende a ser un incentivador del rendimiento académico. Aspectos como la actitud y desempeño, suelen ser poco valorables con respecto a la medición de conocimiento y/o productos, pero que cobran relevancia cuando la objetividad de la evaluación depende de la valoración de la capacidad para resolver problemas, del liderazgo, proactividad, entre otras. El objetivo de este estudio consiste en generar una propuesta para dar la relevancia requerida por la evaluación de actitud y desempeño, a actividades académicas basadas en la generación de soluciones a problemas reales.

Con base a la experiencia en líneas de investigación afines al cómputo ubicuo y a la inteligencia ambiental, se plantea un laboratorio de prácticas con aplicaciones sensibles al contexto, capaz de deducir información de actitud y desempeño relevante, sustentada y medible con evidencias digitales, como principal contribución de esta investigación. 
En la siguiente sección, podrá encontrar una reseña de trabajos de investigación relacionados, posteriormente, encontrará una descripción a detalle de la infraestructura del Entorno Activo, para continuar con la especificación del escenario. Finalmente, las conclusiones y trabajo futuro.

\section{Estado del arte}

Al explorar el concepto de $u$-learning, Burbules (Burbules, 2014) sugiere seis dimensiones para entender de manera más efectiva el concepto de aprendizaje ubicuo: 1) El sentido espacial referente a la presencia de redes "en cualquier lugar", no solo a través de dispositivos móviles sino también en "in situ", que permiten tener acceso continuo a la información; 2) la portabilidad de los dispositivos que pueden llevarse e incluso "vestirse" de manera personal y que pareciera reforzar la expectativa social de que "deberían" estar siempre con uno; 3) la interconexión que permite generar una "inteligencia extensible" mediante el contacto constante con personas que saben o hacen cosas que nosotros no podemos; 4) el sentido práctico por el cual la tecnología desdibuja los límites entre actividades o ámbitos tradicionalmente separados, por ejemplo el trabajo y juego, informal y formal; 5) el sentido temporal referente no solo a la alta disponibilidad sino a la idea del aprendizaje permanente; 6) el sentido de redes y flujos transnacionales y globalizados que implica reconocer las interconexiones básicas entre personas, lugares $\mathrm{y}$ procesos dispares y las maneras en las que estas influyen el aprendizaje personal.

Como menciona Burbules (Burbules, 2014) la brecha tradicional entre contextos formales e informales de aprendizaje está desmoronándose. Los cambios tecnológicos y sociales, culturales e institucionales hacen que el aprendizaje sea una posibilidad continua. Adicionalmente, Velandia-Mesa (VelandiaMesa et al., 2017) señala que los resultados de su estudio revelan que los ambientes $u$ Learning, en comparación con ambientes $e$ Learning, generan diferencias significativas que fortalecen un proceso permanente para aprender por medio de la personalización, adaptación y el aprendizaje situacional. También remarca que existe un desafío debido a que la incorporación de los ambientes ubicuos de aprendizaje requieren una alta inversión en recursos humanos y materiales.
Aunado a esto, (Reyes et al., 2017) en su estudio sobre la motivación en estudiantes de ingeniería civil, obtuvo como resultado que el $73 \%$ de los estudiantes percibió que el uso de la plataforma Moodle y otras técnicas de enseñanza y evaluación los habían motivado de manera significativa, además, un $86 \%$ de estudiantes destacaron que el uso del aprendizaje cooperativo les ayudó a aprender.

En la revisión sistemática realizada por Cárdenas y Peña (Cardenas and Peña, 2018), se examinaron 176 trabajos relacionados con $u$ learning y concluye que unos de los principales retos es crear escenarios apropiados para realizar experiencia de aprendizaje auténticos y envolventes, fomentando las habilidades sociales para explotar la diversidad de estímulos y la carga cognitiva. También señaló la amenaza de lidiar con la volatilidad de la tecnología, la falta de recursos para explorar los avances tanto tecnológicos como educativos y la resistencia al cambio frente a paradigmas novedosos.

Adicionalmente remarca las oportunidades de incorporar el desarrollo masivo de aplicaciones y contenidos para enriquecer las experiencias de aprendizaje a lo largo de la vida de las personas. Finalmente identifica la debilidad respecto a la falta de una línea base teórica, como modelos, arquitecturas, principios de ingeniería, para fundamentar el campo del u-learning.

Existen varios proyectos referentes al $u$ learning, uno de ellos desarrollado por Chin and Chen (Chin and Chen, 2013) en el cual se describe el Mobile Learning Support System (MLSS), orientado a entorno de $u$-learning, que se basaba en el uso de QR codes para facilitar el acceso a multimedia y contenidos significativos del entorno, y también, la localización GPS para identificar la posición física y proveer automáticamente servicios de información de acuerdo al contexto. Otro ejemplo de proyectos de $u$-learning aplicados es el descrito por (Moreno-López et al., 2017) en el que se propone un modelo de aprendizaje ubicuo basado en videos que podían ser desplegados en pantalla de "cualquier lugar" con conectividad a Internet que son accesibles mediante un $Q R$ code, los cuales son generados en un laboratorio de dispositivos y escaneaos por medio de algún dispositivo móvil para reproducir el video. 
En el proyecto LORAMS (Link of RFID and Movies System) Ogata (Ogata et al,. 2009) proporciona soporte a los estudiantes mediante un sistema para compartir y reusar experiencias de aprendizaje ligadas a videos y objetos embebidos, este sistema cuenta con tres fases, la primera es la grabación donde se genera el video que se liga a una etiqueta RFID y se carga en un servidor; la segunda donde por medio de un lector RFID detecta objetos embebidos en el entorno y muestra una lista de videos asociados a dichos objetos, por último, la tercera, el sistema extrae y reproduce el video correspondiente a los objetos detectados y seleccionados por el estudiante. Como resultados, inicialmente, se obtuvo un incremento en el grado de éxito en la realización de las prácticas de un $83.3 \%$ a comparación con un $33.3 \%$ obtenido con solo consultar mediante un buscador WEB.

En su presentación de escenario para enriquecer perfiles digitales de estudiantes, (Martínez-Villaseñor et al., 2014) remarca la necesidad de generar un modelo de usuario en el entorno u-learning para obtener un mejor entendimiento del mismo y proveer servicios de manera personalizada y proactiva, además, puntualiza las problemáticas de un modelo de usuario en los entornos ubicuos, como lo es la evolución, espontaneidad y diversidad de la información y preferencias de los usuarios, lo anterior complicado aún más por aspectos contextuales y de privacidad.

Por otro lado, en el ámbito de las evaluaciones mediante e-learning, (Ehuletche and De Stefano, 2011) durante su investigación de diseño curricular por competencias $\mathrm{y}$ criterios de evaluación para la formación de tutores de e-learning enfoca los instrumentos de evaluación hacia escalas Likert, reportes de participación y entrevistas, y concluyó que el tutor de e-learning requiere habilidades para presencias más frecuentes, para gestionar interacciones y aportes constantes a los alumnos, para guiar la construcción de textos enriquecidos, para gestionar los circuitos feedback, para sostener la ausencia de presencia social con textos enriquecidos con comunicaciones socioemocionales.
En su análisis de la evolución desde $e$ learning hacia el u-learning, Sánchez (Sánchez et al., 2013) pone énfasis en la integración de la evaluación en el proceso de aprendizaje con un sentido formativo y resalta la necesidad de adaptar la evaluación a la demanda de capacitación continua de los estudiantes. Propone entonces el concepto de U-Portafolio para el aprendizaje y evaluación ubicuas, en el cual se refiere a evidencias de ejecuciones y logros obtenidos de los estudiantes en trayectorias de aprendizaje recopilados en cualquier contexto y bajo cualquier modalidad mediante la tecnología. Lo anterior conlleva a que la evaluación del proceso de aprendizaje tenga un enfoque más enriquecedor, posicionándose así en un elemento clave para conseguir un aprendizaje efectivo.

En un estudio sobre el área de Educación Física basado en aprendizaje ubicuo (Gallego-Lema et al., 2017) se usaron dispositivos móviles, geolocalización, realizada aumentada y videos en línea. Para valorar el proceso de aprendizaje se utilizaron, entre otros, la autoevaluación y coevaluación obteniendo beneficios como motivación, mayor interiorización, mayor colaboración y cohesión entre compañeros. En contra parte, se identificaron algunos aspectos adversos como la saturación tecnología y la pérdida de enfoque en las actividades de aprendizaje.

\section{Metodología}

\section{Descripción del Entorno Activo}

AmILab es un Laboratorio que se desarrolla en el ámbito de interfaces de usuario (Human Computer Interfaces) aplicadas hacia un área emergente como lo son los "Entornos Activos" (Active Environments), también llamados "Entornos Inteligentes" (Intelligent Environments, Smart Environments).

Los Entornos Activos integran muchos y variados dispositivos de cómputo, dispersos e integrados de tal manera que pasan desapercibidos a los usuarios (Ubiquitous Computing). Estos dispositivos, son capaces de establecer comunicaciones y de ofrecer servicios a través de novedosas interfaces, ayudando al usuario en sus tareas cotidianas de una manera poco intrusiva y sensible al contexto de la tarea que se está ejecutando. 
El entorno en cuestión se encuentra actualmente en un estado de desarrollo avanzado y funcional, desenvolviéndose como un laboratorio de pruebas que promueve la colaboración en líneas de investigación afines a la inteligencia ambiental y al desarrollo de interfaces de usuario a través de tres escenarios que consisten en una sala de reuniones, una sala de estar, y un aula de clases.

Se ha desarrollado un primer prototipo a través de las siguientes tecnologías: 1) La infraestructura física del entorno, está formada por una red de sensores (presencia, temperatura, iluminación, etc.) y actuadores (switches, etc.) comerciales y estandarizados. Una parte de ellos son dispositivos domóticos X10, algunos otros son tecnología heterogénea (Phidget, Arduino, Intel Galileo). Hay una red adicional Ethernet, encargada del transporte de datos, audio y video generados por las diferentes fuentes disponibles (cámaras IP, webcams, audio streaming, etc.); 2) la integración de nuevos dispositivos no representa ningún problema, solo basta con conectarlo a una de las dos redes (dependiendo de su naturaleza), y la capa de contexto se encargará de ejercer el control sobre el dispositivo y su información. Por ejemplo, la puerta principal del laboratorio fue equipada con un lector RFID. El acceso al laboratorio se hace pasando una tarjeta por el lector, esta envía el código a la capa de contexto donde se realiza la identificación de la persona, procediendo al envío del comando de apertura de puerta.

Para el entorno AmILab-Mx, se propone una capa de contexto que sirve de interfaz entre los diversos dispositivos de cómputo, hacia la integración de un entorno activo (Haya et al., 2004). La capa de contexto empleada, parte de la filosofía de que la representación del mundo se puede hacer a través de un modelo centralizado. Respecto a su implementación, puede hacerse distribuida, proporcionando la suficiente potencia para representar las interacciones complejas de todo lo que convive en el entorno. Además, la capa de contexto proporciona un modelo unificado del mundo, abstrayendo los detalles de comunicación y las diferencias que existen entre los dispositivos del entorno. La implementación del modelo resulta en una estructura de datos global llamada "pizarra" (Englemore and Morgan, 1988).
La pizarra es un repositorio donde se almacena una representación de todo lo que existe en el entorno, incluyendo a los usuarios. Por lo tanto, cada dispositivo, usuario y recurso del entorno activo estará representado mediante una entidad en la pizarra. Además, contiene una representación del flujo de información existente entre los dispositivos físicos (micrófonos, altavoces, cámaras, pantallas, etc.).

La información de la pizarra es utilizada por los diferentes dispositivos para comprender el contexto y adaptarse a este. Cada pizarra es un servidor que puede ser accedido mediante el protocolo cliente-servidor TCP/IP. HTTP ha sido escogido como el protocolo de transporte por su simpleza y amplia cobertura. Para el intercambio de información entre las aplicaciones y la pizarra en el servidor, se emplea el lenguaje XML. La capa de contexto implementada en la estructura de datos llamada pizarra, proporciona el soporte requerido por las aplicaciones. Se han implementado dos interfaces de usuario en el entorno activo: una interfaz gráfica que funciona a través de Internet (Alamán et al., 2003) y que permite controlar los dispositivos de la habitación, la segunda, es un agente de diálogo en lenguaje natural (Montoro et al., 2004) que permite que el usuario interactúe con el entorno. Ambas, son configuradas dinámicamente a través de la información proporcionada por la pizarra.

Agentes basados en reglas que proporcionan los medios para hacer que el entorno se adapte a las necesidades del usuario a través de un control indirecto (García-Herranz et al., 2008). Para lograr este comportamiento interactivo, se ha implementado un sistema de agentes cuyo fin es dotar de un mecanismo mediante el cual los diferentes usuarios puedan comunicar sus preferencias sobre cómo abordar necesidades de comportamiento automático, a fin de modelar el entorno a su gusto.

La arquitectura de este componente de software se basa en un conjunto de módulos independientes entre sí (los agentes), cada uno de los cuales contiene un conjunto de reglas que modelan las necesidades específicas de cada usuario sobre el comportamiento del entorno. Cada regla está constituida por tres elementos (García-Herranz et al., 2008): 
El detonante que define la propiedad de una entidad supervisada y dispuesta en el entorno, responsable de activar la regla. Las condiciones, que especifican las condiciones del contexto sobre las cuales la regla es considerada válida para su ejecución. Y por último la acción, que describe los cambios que deben suceder en el entorno dadas las condiciones del contexto. Así pues, una regla puede expresar que cuando la puerta se abra (el detonante), si la luz está apagada (la condición), se encienda la luz (la acción).

Nuestro laboratorio incorpora diversas tecnologías que además son heterogéneas, desde componentes hardware (tales como sensores, actuadores, electrodomésticos, webcams, etc.) hasta componentes de software, tales como reconocedores y sintetizadores de voz. Todos estos componentes tienen que ser integrados y controlados de la misma manera, es decir, usando las mismas interfaces de usuario. Por ejemplo, un usuario debe ser capaz de encender un servidor de música de una manera tan simple y fácil como lo es apagar o encender las luces de una habitación.

Por otra parte, la interacción del usuario debe mantenerse tan flexible como sea posible, esto se puede lograr haciendo uso de múltiples modalidades, servicios web, voz, tacto, y tantos otros como sean las preferencias y capacidades del usuario. Sin embargo, la configuración de un entorno activo es de por si altamente dinámica, por lo que cambia de un entorno a otro. Se agregan componentes, se eliminan o bien se mantienen detenidos en sus funciones durante bastante tiempo, y las interfaces de usuario deben ser capaces de conocer estos cambios.

Un agente de diálogo en lenguaje natural es una buena alternativa para controlar un entorno activo (Montoro et al., 2004). La importancia de esta interfaz de diálogos radica en su manera de apoyarse en la información contextual almacenada por la pizarra, haciendo posible establecer una conversación coherente relacionada con el control de los dispositivos y la información de los usuarios.

La interfaz ejecuta varios diálogos relativos al entorno que compiten por ser el más idóneo para la conversación actual.
Un supervisor de diálogos, tiene la tarea de elegir el diálogo más adecuado de acuerdo a la entrada de datos proporcionada por el usuario (proporcionada por el reconocedor de voz) y la información contextual proveniente de la pizarra. Además, el supervisor de diálogos tiene la tarea adicional de activar y desactivar diálogos en función de si tienen o no sentido.

Cada diálogo, se centra en una tarea específica, por ejemplo, el diálogo de las luces se encarga de controlar el estado encendido o apagado de las lámparas de una habitación. Esto es, el diálogo tiene la capacidad de leer y escribir sobre los valores de las lámparas almacenadas en la pizarra.

Debido a que la fase de reconocimiento de voz no es del todo exacta, la información de contexto proveniente de la pizarra toma un rol muy importante en las decisiones del supervisor, esto es, cada sentencia que proviene del usuario, puede venir con ambigüedades, y el supervisor las resuelve empleando la información de la pizarra. Otra opción, es ofrecer soluciones al usuario dependientes del contexto. Por ejemplo, si el reconocedor de voz solo ha detectado con precisión la palabra luz, el supervisor podrá corroborar el estado de las luces. Si estas están apagadas, directamente puede ofrecer la opción de encenderlas.

El laboratorio cuenta con una interfaz gráfica que es desplegada por cualquier navegador a través de un applet y desarrollada para controlar todos los dispositivos y electrodomésticos del entorno activo. Esta aplicación se puede considerar como una vista parcial de la información almacenada en la estructura de datos llamada pizarra.

Al iniciar la aplicación, hace una lista de las habitaciones del laboratorio y para cada habitación, genera un mapa que incluye una representación gráfica de cada dispositivo que se encuentra físicamente en el entorno, así como su localización. Cada vez que el usuario hace clic con el ratón sobre la imagen de un dispositivo, aparece una interfaz (panel de control) que controla el funcionamiento de ese dispositivo (por ejemplo: si hace clic sobre una lámpara, aparece un botón que la enciende o la apaga). 
Con el objetivo de proporcionar una nueva manera de interacción entre los dispositivos del entorno con el uso de dispositivos móviles, se generó una interfaz de usuario a través de códigos de dos dimensiones y un lector genérico en los dispositivos móviles. La interacción puede ser de dos maneras, un actuado directo que consiste de poner etiquetas a electrodomésticos cuya actuación se reduce a un estado de encendido o apagado, tal es el caso de las luces de las habitaciones. En la segunda, la lectura de un código conduce a un menú de actuado, tal es el caso del televisor, o de un entorno con todos sus electrodomésticos.

\section{Descripción del Escenario}

Gran parte de los beneficios documentados por los diversos proyectos de u-learning, se encuentran en la integración de escenarios externos (outdoor), adaptados para interactuar con los usuarios mediante los dispositivos adecuados y contenido digital (MuñozCristóbal et al., 2014) (Tabuenca et al., 2014). En el extremo opuesto, tradicionalmente las actividades educativas se realizan en un entorno controlado (indoor), donde es prioritario un escenario propicio para la interacción (Felix et al., 2017), con propincuidad espacial que fomente la colaboración (Kraut et al., 2014). AmILab, es un Entorno Activo controlado donde se han remplazado las butacas convencionales por cuatro mesas de trabajo. Cada mesa está equipada con una videocámara en la parte superior que almacenará solo la actividad que se desarrolle en la superficie de la mesa. Cabe mencionar que las cámaras están también integradas a la capa de contexto, por lo que se puede actuar sobre sus propiedades desde cualquiera de las interfaces de usuario.

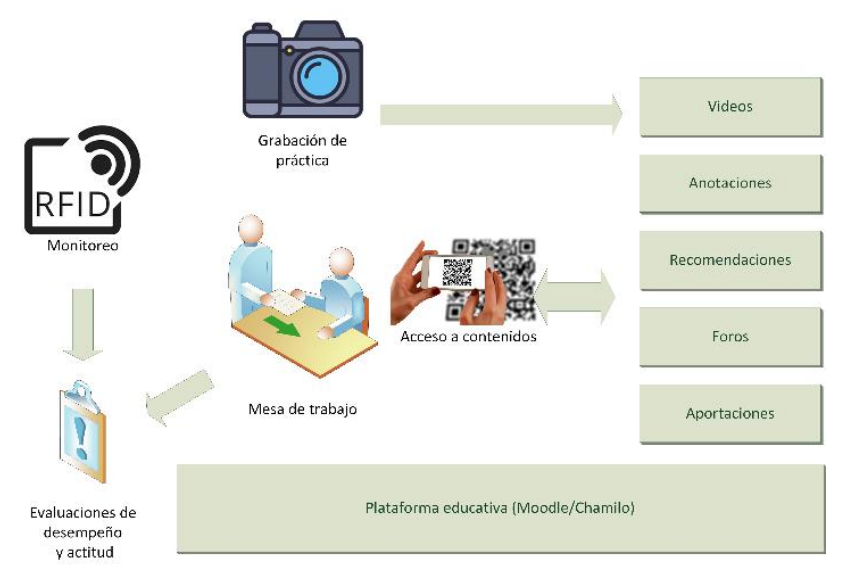

Figura 1 Arquitectura de escenario de u-learning en AmILabMX
Adicional a la infraestructura que propone el Entorno de Aprendizaje Ubicuo, es indispensable formular una estrategia para la generación de conocimiento. En el ámbito universitario, el desempeño académico de algunas materias está relacionado directamente con las habilidades para resolver problemas prácticos, es decir, el saber hacer.

El aprendizaje entre pares propone el uso de estrategias de enseñanza y aprendizaje en las cuales los estudiantes aprenden unos de otros sin la intervención inmediata de un maestro (Boud et al., 1999). La generación de videos cortos, puede resultar en una buena estrategia de enseñanza y aprendizaje, que aplicando la teoría de la Zona de Desarrollo Próximo (Moll, 1990), permita alcanzar un proceso de simbiosis entre los integrantes a partir de crear, discutir y compartir conceptos y ejercicios.

Así pues, la implementación de la estrategia para la generación del conocimiento tiene los siguientes requerimientos:

Un Entorno Activo capaz de interactuar con el contexto de una manera poco intrusiva y bajo el paradigma del cómputo ubicuo.

- El entorno proveerá la infraestructura para elaborar los productos de trabajo, que consisten en la elaboración de material videográfico de corta duración. Los productos deben comprender tres etapas bien diferenciadas: Resumen, Contenido y Conclusión.

- $\quad$ La generación de los productos, deberá utilizar la infraestructura del Entorno Activo, la cual comprenderá una plataforma para la administración de los objetos de aprendizaje, clasificación y recomendación.

Una parte valorable de la propuesta consiste en generar una actitud proactiva de los estudiantes. La plataforma permitirá la consulta de contenidos desde remoto, a partir de dispositivos móviles. 
La evaluación formativa tiene como intención generar una retroalimentación valorable sobre el desempeño de un estudiante, que permita mejorar y acelerar el aprendizaje (Sadler, 1998). Black y William (Black and William, 1998) concluyeron que si la retroalimentación obtenida de una evaluación formativa está relacionada con buenas prácticas de enseñanza-aprendizaje, y proporciona información sobre cómo mejorar el rendimiento, tendría un efecto positivo en los estudiantes.

La evaluación debe darse en base a cuatro criterios: Conocimiento, Producto, Desempeño y Actitud (véase la tabla 1). La mayoría de los sistemas de E-Learning y $U$ Learning hacen énfasis en la evaluación del conocimiento y producto. En el escenario propuesto, note que no se hace referencia a la evaluación del conocimiento, ni al mecanismo o métrica para evaluar el producto, asumimos que ambas son responsabilidad única del docente, y que la plataforma ofrecerá los mecanismos para una evaluación objetiva.

\begin{tabular}{|c|c|c|}
\hline $\begin{array}{l}\text { Contenidos } \\
\text { educativos }\end{array}$ & $\begin{array}{l}\text { Técnicas de } \\
\text { evaluación }\end{array}$ & Instrumentos \\
\hline $\begin{array}{l}\text { Conceptual } \\
\text { (Conocimiento) }\end{array}$ & Orales/Escritos & $\begin{array}{l}\text { Cuestionarios. } \\
\text { Entrevistas. }\end{array}$ \\
\hline $\begin{array}{l}\text { Procedimental } \\
\text { (Producto) }\end{array}$ & $\begin{array}{l}\text { Escritos, } \\
\text { Manipulativos }\end{array}$ & $\begin{array}{l}\text { Portafolio de } \\
\text { evidencias. } \\
\text { Rúbricas. } \\
\text { Listas de cotejo. } \\
\text { Escalas de rango. }\end{array}$ \\
\hline $\begin{array}{l}\text { Procedimental } \\
\text { (Desempeño) }\end{array}$ & $\begin{array}{l}\text { Observación, } \\
\text { Manipulativos }\end{array}$ & $\begin{array}{l}\text { Coevaluaciones. } \\
\text { Registros de } \\
\text { actividad. } \\
\text { Contribuciones. } \\
\text { Estadísticas. }\end{array}$ \\
\hline $\begin{array}{l}\text { Actitudinal } \\
\text { (Actitud) }\end{array}$ & $\begin{array}{l}\text { Escritos, } \\
\text { Observación }\end{array}$ & $\begin{array}{l}\text { Coevaluaciones. } \\
\text { Reportes de } \\
\text { comportamiento. }\end{array}$ \\
\hline
\end{tabular}

Tabla 1 Relación de contenidos educativos y técnicas de evaluación u-learning propuestas para el modelo de competencias aplicado en el sistema del TecNM

Como antecedente, la propuesta contempla un laboratorio de prácticas en donde el estudiante pueda acudir y trabajar sin presencia del docente. La meta del escenario de trabajo, consiste en realizar una aportación respecto a la evaluación de Actitud y Desempeño.
¿Cómo se podría hacer una evaluación sin la observación directa de los estudiantes? Para dar respuesta a la pregunta anterior, se debe confiar en la infraestructura del Entorno Activo y en sus aplicaciones sensibles al contexto. Algunos criterios a evaluar como parte del Desempeño son:

- La asistencia, obtenida de una aplicación que obtenga el perfil del estudiante a partir de su identificación con un dispositivo RFID, por ejemplo.

- Evaluación de la participación, valor obtenido del cumplimiento de roles y generación de entregables dentro del equipo de trabajo.

Contribuciones individuales, que comprende la medición de la actitud proactiva del estudiante, que puede ser, recomendación de videos con la solución factible e incluso, notificación de errores o procedimientos equivocados en los que ya no deben incurrir otros equipos.

Estadísticas de actividad, obtenidas de la interacción con la plataforma.

En lo que respecta a la Actitud, una alternativa para su cuantificación puede ser acudir a una evaluación por pares, en donde los integrantes del equipo, e incluso entre otros equipos, puedan emitir una retroalimentación acerca del liderazgo, proactitud, trabajo global del equipo, infracciones al reglamento y respeto en la comunicación.

\section{Conclusiones}

Black y William (Black and William, 1998) reconocen como esencial y necesario el rol del profesor, como mediador entre un conjunto de conocimientos enriquecidos con habilidades para enseñar, y el alumno. La función del docente básicamente se reduce a hacer el aprendizaje más eficiente. Esta perspectiva de la enseñanza aplica cuando el maestro acepta esta responsabilidad, derivado de esto, gran parte del éxito del proyecto depende, no de la constante presencia del docente, sino de un esfuerzo y responsabilidad mayor al que haría en un esquema de enseñanza tradicional, para generar el curso e incentivar las actividades de aprendizaje, cuya participación los estudiantes requiere planificación, seguimiento y retroalimentación. 
El aporte de nuestro trabajo de investigación consiste un escenario de $u$ learning que promueve el aprendizaje basado en la resolución de problemas en un entorno activo, que integra mecanismos de evaluación de aspectos orientados a la Actitud y Desempeño. Aún y cuando no es posible anticipar su objetividad, se plantea una propuesta para su medición sustentada y basada en evidencias digitales.

\section{Trabajo futuro}

Se permanece a la espera de los primeros resultados de la implementación de cursos bajo el esquema de $u$-learning, con el objetivo de ver la relación de estos con una posible valoración de calidad y efectividad del aprendizaje.

\section{Agradecimientos}

Este estudio fue posible gracias a la financiación del Tecnológico Nacional de México a través del proyecto "Fortalecimiento de Cuerpos Académicos (IDCA 28030)" en el marco de la convocatoria 2017 otorgado al ITESZAN-CA-2. Así mismo, al apoyo del Instituto Tecnológico Superior Zacatecas Norte y el Tecnológico Nacional de México.

\section{Referencias}

Acosta, M. and Armendáriz, G. (2012). Modelo educativo para el siglo XXI: Formación y desarrollo de competencias profesionales. Dirección General de Educación Superior tecnológica. México. DF.

Alamán, X., Cabello, R., Gómez-Arriba, F., Haya, P., Martínez, A., Martínez, J., and Montoro, G. (2003). Using context information to generate dynamic user interfaces. In Proceedings of the Tenth International Conference on Human-Computer Interaction, 2, 345-349.

Black, P. and Wiliam, D. (1998). Assessment and classroom learning. Assessment in Education: principles, policy \& practice, 5(1), 7-74.

Boud, D., Cohen, R., and Sampson, J. (1999). Peer learning and assessment. Assessment \& evaluation in higher education, 24(4):413-426.
Burbules, N. C. (2014). Los significados de "aprendizaje ubicuo". Education Policy Analysis Archives, 22:1-10.

Cárdenas-Robledo, L. A., \& Peña-Ayala, A. (2018). Ubiquitous learning: A systematic review. Telematics and Informatics, 35(5), 1097-1132.

Chin, K.-Y., \& Chen, Y.-L. (2013). A Mobile Learning Support System for Ubiquitous Learning Environments. Procedia - Social and Behavioral Sciences, 73, 14-21.

Ehuletche, A. M. \& De Stefano, A. (2011). Evaluación de las competencias para la formación de tutores de E-Learning. RIED. Revista Iberoamericana de Educación a Distancia, 14(1), 75-86.

Englemore, R. and Morgan, A. (1988). Blackboard Systems; Edited by Robert Engelmore, Tony Morgan (the Insight Series in Artificial Intell.) Addison-Wesley Longman Publishing Co., Inc.

Felix, V. G., Mena, L. J., Ostos, R., and Maestre, G. E. (2017). A pilot study of the use of emerging computer technologies to improve the effectiveness of reading and writing therapies in children with down syndrome. British Journal of Educational Technology, 48(2), 611-624.

Gallego Lema, V., Muñoz Cristóbal, J. A., Arribas Cubero, H. F., Rubia Avi, B., et al. (2017). La orientación en el medio natural: aprendizaje ubicuo mediante el uso de tecnología. Movimento, (ESEFID/UFRGS), 23(2), 755 .

García-Herranz, M., Haya, P. A., Esquivel, A., Montoro, G., and Alamán, X. (2008). Easing the smart home: Semi-automatic adaptation in perceptive environments. J. UCS, 14(9), 15291544.

Haya, P. A., Montoro, G., and Alamán, X. (2004). A prototype of a context-based architecture for intelligent home environments. In OTM Confederated International Conferences" On the Move to Meaningful Internet Systems", pages 477-491. Springer. 
Kraut, R. E., Egido, C., and Galegher, J. (2014). Patterns of contact and communication in scientific research collaborations. In Intellectual teamwork, pages 163-186. Psychology Press.

Martínez-Villaseñor, M. de L., GonzálezMendoza, M., \& Danvila Del Valle, I. (2014). Enrichment of Learner Profile with Ubiquitous User Model Interoperability. Computación y Sistemas, 18(2), 359-374.

Moll, L. C. (1990). La zona de desarrollo próximo de vygotski: Una reconsideración de sus implicaciones para la enseñanza. Infancia y aprendizaje, 13(51-52), 247-254.

Montoro, G. et al. (2004). Spoken interaction in intelligent environments: a working system, advances in pervasive computing, eds. Austrian Computer Society (OCG).

Moreno López, G. A., Jiménez Builes, J. A., \& Ramírez Monsalve, E. J. (2017). Ubiquitous learning model based on platforms of multiscreen TV (uLMTV). DYNA, 84(203), 160169.

Muñoz-Cristóbal, J. A., Jorrín-Abellán, I. M., Asensio-Pérez, J. I., Martínez- Mones, A., Prieto, L. P., and Dimitriadis, Y. (2014). Supporting teacher orchestration in ubiquitous learning environments: A study in primary education. IEEE Transactions on Leaning Technologies, 8(1), 83-97.

Ogata, H., Matsuka, Y., El-Bishouty, M. M., and Yano, Y. (2009). Lorams: linking physical objects and videos for capturing and sharing learning experiences towards ubiquitous learning. International Journal of Mobile Learning and Organisation, 3(4):337-350.

Ogata, H. and Yano, Y. (2004). Context-aware support for computer-supported ubiquitous learning. In The 2nd IEEE International Workshop on Wireless and Mobile Technologies in Education, 2004. Proceedings., pages 27-34. IEEE.

Reyes, E., Enfedaque, A., \& Gálvez, J. C. (2017). Initiatives to foster engineering student motivation: A case study. Journal of Technology and Science Education, 7(3), 291312.
Sadler, D. R. (1998). Formative assessment: Revisiting the territory. Assessment in education: principles, policy \& practice, $5(1), 77-84$.

Sánchez, R., Mallado, C., González-Piñal, R. (2013). Cambios pedagógicos y sociales en el uso de las TIC: U-learning y u-Portafolio. Revista Electrónica de Investigación y Docencia (REID), 10:7-20.

Tabuenca, B., Kalz, M., Ternier, S., and Specht, M. (2014). Stop and think: Exploring mobile notifications to foster reflective practice on meta-learning. IEEE Transactions on Learning Technologies, 8(1):124-135.

Velandia-Mesa, C., Serrano-Pastor, F.-J., \& Martínez-Segura, M.-J. (2017). Formative Research in Ubiquitous and Virtual Environments in Higher Education. Comunicar, 25(51), 09-18. 